\title{
Thiamin biofortification of crops
}

Aymeric Goyer ${ }^{a, b}$

${ }^{\text {a }}$ Department of Botany and Plant Pathology, Oregon State University, Corvallis, OR 97330, United States of America

${ }^{\mathrm{b}}$ Hermiston Agricultural Research and Extension Center, Oregon State University, Hermiston, OR 97838, United States of America

\section{Corresponding author:}

Aymeric Goyer

aymeric.goyer@oregonstate.edu

(C) 2016. This manuscript version is made available under the Elsevier user license http://www.elsevier.com/open-access/userlicense/1.0/ 


\begin{abstract}
Thiamin is essential for human health. While plants are the ultimate source of thiamin in most human diets, staple foods like white rice have low thiamin content. Therefore, populations whose diets are mainly based on low-thiamin staple crops suffer from thiamin deficiency. Biofortification of rice grain by engineering the thiamin biosynthesis pathway has recently been attempted, with up to fivefold increase in thiamin content in unpolished seeds. However, polished seeds that retain only the starchy endosperm had similar thiamin content than that of non-engineered plants. Various factors such as limited supply of precursors, limited activity of thiamin biosynthetic enzymes, dependence on maternal tissues to supply thiamin, or lack of thiamin stabilizing proteins may have hindered thiamin increase in the endosperm.
\end{abstract}

\title{
Introduction
}

Thiamin (vitamin $\mathrm{B}_{1}$ ), in its diphosphate form (ThDP), functions as a cofactor for key enzymes of carbohydrates and amino acid metabolism in all living organisms [1,2]. While plants, fungi and bacteria can synthesize thiamin de novo, humans cannot and must obtain it from food. Although plant foods are a major source of thiamin, some staple crops like rice contain relatively low amounts of thiamin. Industrialized milling of the rice grain that removes the outer layers of the seed, i.e. the pericarp, testa, nucellus, and aleurone layer, as well as the embryo (Figure 1), further depletes the grain's thiamin content [3]. The remaining white starchy endosperm that is widely consumed worldwide contains very small amounts of thiamin. Populations whose diets are mostly based on low thiamin and high carbohydrates foods like white rice commonly suffer from thiamin deficiency [4,5]. Symptoms of severe thiamin deficiency include degeneration in the nerves and heart, a disease known as beriberi, which can be fatal. Severe cases of thiamin deficiency are rare in countries like the United States where programs to fortify food with thiamin have been implemented [6]. However, marginal thiamin deficiencies are still common and often remain undiagnosed because of the lack of obvious clinical symptoms $[7,8]$.

Thiamin biofortification of staple crops that have low thiamin content is an attractive strategy to avert thiamin deficiency [9]. In the past two years, the first efforts to biofortify food crops with thiamin have been reported $[10,11]$. These attempts were feasible because of the tremendous progress in our understanding of thiamin metabolism in plants in recent years. The availability of complete plant genome sequences has enabled the identification and cloning of several genes involved in thiamin biosynthesis, transport, and salvage [12,13], and the biochemical characterization of the corresponding proteins. Regulatory elements have also been discovered. This has provided the foundation for the first thiamin engineering strategies. Yet, many aspects of thiamin metabolism remain poorly understood, which hinders the implementation of rational thiamin engineering strategies. In this review, I will first summarize the current knowledge about thiamin biosynthesis in plants. Next, I will highlight efforts that have been made in the last two years to increase thiamin content in plants by genetic 
engineering. Finally, I will address the specific issue of the seed and discuss future potential strategies to further increase thiamin content in major food grains.

\section{The de novo thiamin biosynthetic pathway in plants}

Thiamin is made of a pyrimidine and thiazole moiety that are synthesized in the chloroplast (Figure 2). Today, most genes that are involved in de novo thiamin biosynthesis have been identified and some regulatory features of the pathway have been characterized. The first step in the synthesis of the pyrimidine moiety involves 4-amino-2-methyl-5-hydroxymethylpyrimidine phosphate (HMP-P) synthase (THIC) that utilizes 5-aminoimidazole ribonucleotide (AIR) and $S$ adenosylmethionine (SAM) as substrates to form HMP-P [14,15] (Figure 2). An important element of THIC regulation is the presence of a riboswitch in the 3' untranslated region (UTR) of THIC mRNA precursor (Figure 2) [16-20]. This riboswitch is a sensor of ThDP concentration in the cell that controls THIC mRNA stability and thereby ThDP production. The first step in the synthesis of the thiazole moiety involves 4-methyl-5- $\beta$-hydroxyethylthiazole phosphate (HET-P) synthase (THI1) [21] that utilizes nicotinamide adenine dinucleotide (NAD) and glycine as substrates and supplies a sulfide from a conserved cysteine residue to form an adenylated thiazole intermediate (ADT) [22-24]. ADT is then hydrolyzed to HET-P but the NUDIX hydrolase responsible for this step has not been characterized. A bifunctional HMP-P kinase/thiamin monophosphate (ThMP) pyrophosphorylase (TH1) phosphorylates HMP-P to HMP-PP and then condenses HMP-PP to HET-P to form ThMP [25,26]. ThMP is then dephosphorylated to thiamin by a specific haloacid dehalogenase (HAD) family phosphatase (TH2) that is directed to the cytosol or mitochondria (AD Hanson, personal communication); other HAD phosphatases may also contribute to ThMP dephosphorylation [27]. Thiamin is then pyrophosphorylated to ThDP by ThDP kinases (TDPKs) that are located in the cytosol in Arabidopsis [28]. ThDP serves as a cofactor in enzymatic reactions in the cytosol, chloroplast, and mitochondria. One transporter has so far been characterized for ThDP transport to organelles [29].

\section{Engineering of the thiamin biosynthesis pathway}

Bypassing the control exerted by the THIC riboswitch is a rational strategy to increase thiamin content. Arabidopsis plants expressing THIC with a deficient riboswitch in a THIC knockdown background had up to 1.6- and 1.7-fold increase in total leaf and seed thiamin content, respectively [30]. Similar increases in total thiamin content of leaves were reported when THIC was under the control of the Arabidopsis UBIQUITIN(UBI)-1 promoter or the CAULIFLOWER MOSAIC VIRUS (CaMV) 35S promoter and terminators which do not contain a riboswitch [15,30]. Although this approach produced increases in thiamin content, these were relatively modest, suggesting that the thiazole moiety of thiamin may be limiting in plants overexpressing THIC and that both branches of the thiamin biosynthetic pathway should be engineered to obtain 
larger increase in total thiamin pools. The first validation of this hypothesis came from in vitro feeding studies in Arabidopsis [31]. Wild-type Arabidopsis seedlings that were grown on basal salt medium supplemented with both HMP and HET had thiamin levels eightfold higher than that of the control while thiamin levels in seedlings supplemented with HMP or HET alone remained unchanged.

In the last two years, biofortification per se has been attempted in Arabidopsis and rice by overexpressing THIC and THII alone or together under the control of strong constitutive promoters and terminators with no riboswitch [10,11]. Rice plants expressing THIC under the control of the maize UBI promoter and the NOPALINE SYNTHASE terminator did not contain higher amounts of any thiamin forms than those of the control. Similarly, Arabidopsis and rice plants expressing THII under the control of the CaMV35S promoter or the maize UBI promoter, respectively, and terminators which do not contain a riboswitch, did not accumulate thiamin in higher amounts than the control [10,11]. However, when Arabidopsis and rice plants that express THIC or THII transgenes alone were crossed to generate plants that overexpress both transgenes, total thiamin levels increased up to 3.4- and 2.5-fold in Arabidopsis and rice leaves, respectively, and up to 2.6- and 5-fold in Arabidopsis and rice unpolished seeds, respectively (Table 1). These results show that engineering of both pyrimidine and thiazole branches of the thiamin biosynthesis pathway is necessary in order to increase the thiamin content of leaves and seeds to higher amounts than those found in plants engineered for either branch.

\section{Bottlenecks in thiamin biosynthesis}

One potential limiting factor to further thiamin accumulation in plants engineered for both $T H I 1$ and THIC is a limiting supply of precursors. Three thiamin vitamers are typically present in plant leaves: thiamin, ThMP, and ThDP, ThDP making over $90 \%$ of the total thiamin content in wild type plants. In seeds, thiamin is stored in its unphosphorylated form that constitutes over $95 \%$ of the total thiamin content. In leaves of Arabidopsis and rice plants overexpressing both THIC and $T H I 1$, thiamin levels increased over sixfold comprising up to $45 \%$ of the total thiamin pool, ThDP levels increased up to twofold comprising at least $50 \%$ of the total thiamin content, but ThMP pools remained very low, accounting for about $4 \%$ of the total thiamin pool $[10,11]$. This suggests that pools of some precursors of thiamin biosynthesis were exhausted. Therefore, combining overexpression of genes that are involved in precursors, e.g. NADC in NAD biosynthesis [32], and thiamin biosynthesis may be a strategy to further increase thiamin levels.

Limited activities of thiamin biosynthesis enzymes are also potential bottlenecks.

Although THIC and THII transcript levels increased substantially in Arabidopsis and rice plants overexpressing THIC or THII [10,11], it is possible that increases in THIC and THI1 enzymatic activities were modest and not sufficient to allow higher increases in thiamin production. This could be due to limitations in factors which these enzymes rely upon. For instance, both THIC and THI1 activities may be dependent on reduction by thioredoxins as proteomics experiments have suggested $[33,34]$. Moreover, the supply of sulfur for THIC's iron-sulfur cluster is 
dependent on a cysteine desulfurase $[14,35,36]$. THI1 is a single turnover protein that loses its functionality after sacrificing a cysteine residue during reaction $[24,36]$, which implies that a high demand of energy is necessary for THI1 protein synthesis to catalyze one single step of thiamin biosynthesis. In addition, ThMP synthase activity of TH1 could be inhibited by excess HMP-PP in THIC-overexpressing plants as was shown for recombinant TH1 from maize [25]. Protein quantification and enzymatic activity measurements will be necessary to confirm these hypotheses.

\section{The specific issue of the endosperm and potential engineering strategies}

Although engineered rice plants that overexpressed THIC and THII under the control of the constitutive $U B I$ promoter produced seeds with thiamin content up to fivefold greater than that of the control [11], thiamin content increased only up to 1.3-fold in the endosperm, suggesting that the endosperm has little ability to produce and/or store thiamin. While THIC and THII are expressed in the early stages of endosperm development in maize, THIC expression decreases between 14 and 30 days post pollination [13], suggesting that the endosperm's ability to synthesize thiamin de novo is lost in the later stages of development (Figure 1). Therefore, the modest increase of the thiamin content in the endosperm of plants constitutively overexpressing THIC indicate that constituents of the endosperm which THIC relies upon for pyrimidine synthesis, e.g. precursors, are missing in the maturing endosperm. This is in accordance with the suggestion by Guan et al. [13] that part of the endosperm's thiamin content may be obtained by coupling de novo thiazole biosynthesis with pyrimidine salvage (Figure 1). Measurements of thiazole and pyrimidine in the endosperm of the overexpressor lines should help validate these hypotheses, but analytical methods are currently missing.

A large proportion of the endosperm's thiamin pool may actually be supplied from maternal tissues. In vitro radiolabeling feeding experiments showed that thiamin can be transported from maternal tissues (cob) into the endosperm of maize seed [37], and over $90 \%$ of the total thiamin content may come from maternal tissues in developing Arabidopsis seeds $[1,14]$. Therefore, increasing transport of thiamin from maternal tissues (e.g. leaves, hull, pericarp) into the endosperm is a potential strategy to increase the endosperm's thiamin content. A thiamin transporter that is involved in long-distance transport of thiamin from leaves that biosynthesize thiamin to roots that do not biosynthesize thiamin was recently characterized [38]. Similar transporters that supply thiamin from source tissues to the endosperm will need to be identified in order to implement such strategies.

Another limitation to further increasing thiamin in the endosperm (and other tissues or organs) may be the lack of stability of thiamin. Thiamin is extremely prone to chemical or enzymatic damage [12,39-41]. Preemption of damage and salvage mechanisms in thiamin metabolism have recently been described [42-44], but it remains unclear how much these contribute to the homeostasis of thiamin pools in the endosperm. In addition, seeds of many plant species $[2,45]$ synthesize thiamin-binding proteins (TBPs) that may protect thiamin from 
damage. Several studies have reported a close relationship between the increase in thiamin content and the increase in TBP activity during seed maturation [46-49]. Most TBP activity was found in rice bran and little activity was detected in milled rice [45,50,51]. Immunolocalization experiments have shown that TBPs are localized in the aleurone layer of wheat seeds [49]. These data indicate that the endosperm lacks the capability of retaining thiamin during seed maturation and storage, and suggests that expressing TBP in the endosperm may increase the endosperm's thiamin content.

\section{Conclusions}

Recent progress in our understanding of thiamin metabolism in plants has led to the first attempts in the thiamin biofortification of staple crops in the past two years. Overexpression of two genes that are involved in thiamin biosynthesis increased thiamin content up to fivefold in unpolished rice seeds. However, there was very little increase in thiamin content in the starchy endosperm, the tissue remaining after removal of the grain's outer layers during milling to produce white rice. Factors limiting thiamin increase in the endosperm of engineered plants may be a limited supply of thiamin precursors and/or a lack of increased activity of thiamin biosynthetic enzymes. In addition, the endosperm may be a sink tissue that mostly relies on import of thiamin from source tissues. Finally, the endosperm seems to lack thiamin binding proteins that may be essential for thiamin stability. Future research should investigate the divisions of labor in thiamin metabolism in seed tissues and develop tissue-specific metabolic models [52] in order to design rational engineering strategies for thiamin biofortification of the rice endosperm, one of the main food source for billions of people worldwide.

\section{Acknowledgements}

This work was supported by the Bill \& Melinda Gates Foundation, Seattle, WA [grant number OPP1067940].

\section{References}

Papers of particular interest, published within the period of review, have been highlighted as:

- of special interest

•• of outstanding interest

1. Goyer A: Thiamine in plants: aspects of its metabolism and functions. Phytochemistry 2010, 71:1615-1624.

2. Rapala-Kozik M: Vitamin $B_{1}$ (Thiamine): A cofactor for enzymes involved in the main metabolic pathways and an environmental stress protectant. In Biosynthesis of vitamins in plants: Vitamins A, B1, B2, B3, B5. Edited by Rebeille F, Douce R. Academic Press Ltd-Elsevier Science Ltd; 2011:37-91. 
3. Juliano BO: Rice in human nutrition. FAO Food and Nutrition Series No. 26; 1993:1-162.

4. WHO: Thiamine deficiency and its prevention and control in major emergencies. Micronutrients Series, World Health Organization 1999, WHO/ND/99.13:1-45.

5. Whitfield KC, Karakochuk CD, Liu YZ, McCann A, Talukder A, Kroeun H, Ward M, McNulty H, Lynd LD, Kitts DD, et al.: Poor thiamin and riboflavin status is common among women of childbearing age in rural and urban Cambodia. $J$ Nutr 2015, 145:628-633.

6. Backstrand JR: The history and future of food fortification in the United States: A public health perspective. Nutr Rev 2002, 60:15-26.

7. Lonsdale D: A review of the biochemistry, metabolism, and clinical benefits of thiamin (e) and its derivatives. Evidence-based Complementary and Alternative Medicine 2006, 3:49-59.

8. Rao SN, Chandak GR: Cardiac beriberi: Often a missed diagnosis. J Trop Pediatr 2010, 56:284-285.

9. Fitzpatrick TB, Basset GJC, Borel P, Carrari F, DellaPenna D, Fraser PD, Hellmann H, Osorio S, Rothan C, Valpuesta V, et al.: Vitamin deficiencies in humans: Can plant science help? Plant Cell 2012, 24:395-414.

10. Dong W, Stockwell VO, Goyer A: Enhancement of thiamin content in Arabidopsis thaliana by metabolic engineering. Plant Cell Physiol 2015, 56:2285-2296.

- $\quad$ This study is the first report of engineering the thiamin biosynthetic pathway in plants. The authors show that a minimum of two thiamin biosynthetic genes, thiC and thil, must be overexpressed to increase thiamin content in Arabidopsis leaves and seeds.

11. Dong W, Thomas N, Ronald PC, Goyer A: Overexpression of thiamin biosynthesis genes in rice increases leaf and unpolished grain thiamin content but not resistance to Xanthomonas oryzae pv. oryzae. Frontiers in Plant Science 2016, 7.

•- $\quad$ This study is the first report of engineering the thiamin biosynthetic pathway in a staple crop. The authors show that rice plants that express the two thiamin biosynthesis genes thiC and thil under the control of constitutive promoters produce seeds with thiamin level up to fivefold greater than that of the non-engineered plants, but the increase in thiamin content occurs mostly in the outer layers of the grain, not in the endosperm.

12. Colinas M, Fitzpatrick TB: Natures balancing act: examining biosynthesis de novo, recycling and processing damaged vitamin B metabolites. Curr Opin Plant Biol 2015, 25:98-106.

•- $\quad$ This review provides an excellent overview of recent research developments in thiamin metabolism in plants.

13. Guan JC, Hasnain G, Garrett TJ, Chase CD, Gregory JF, Hanson AD, McCarty DR: Divisions of labor in the thiamin biosynthetic pathway among organs of maize. Frontiers in Plant Science 2014, 5:1-11. 
- $\quad$ This study reports divergent expression pattern of thiamin biosynthesis, salvage, and transport genes among organs of maize, and highlights different strategies for thiamin acquisition among organs, a concept that the authors introduce as divisions of labor.

14. Raschke M, Burkle L, Muller N, Nunes-Nesi A, Fernie AR, Arigoni D, Amrhein N, Fitzpatrick TB: Vitamin $B_{1}$ biosynthesis in plants requires the essential iron sulfur cluster protein, THIC. Proc Natl Acad Sci U S A 2007, 104:19637-19642.

15. Kong D, Zhu Y, Wu H, Cheng X, Liang H, Ling HQ: AtTHIC, a gene involved in thiamine biosynthesis in Arabidopsis thaliana. Cell Res 2008, 18:566-576.

16. Wachter A, Tunc-Ozdemir M, Grove BC, Green PJ, Shintani DK, Breaker RR: Riboswitch control of gene expression in plants by splicing and alternative 3 ' end processing of mRNAs. Plant Cell 2007, 19:3437-3450.

17. Wachter A: Riboswitch-mediated control of gene expression in eukaryotes. RNA Biol. 2010, 7:1-9.

18. Bocobza S, Adato A, Mandel T, Shapira M, Nudler E, Aharoni A: Riboswitch-dependent gene regulation and its evolution in the plant kingdom. Genes Dev 2007, 21:28742879.

19. Sudarsan N, Barrick JE, Breaker RR: Metabolite-binding RNA domains are present in the genes of eukaryotes. RNA 2003, 9:644-647.

20. Bocobza SE, Aharoni A: Switching the light on plant riboswitches. Trends Plant Sci. 2008, 13:526-533.

21. Ribeiro A, Praekelt U, Akkermans AD, Meacock PA, van Kammen A, Bisseling T, Pawlowski K: Identification of agthi1, whose product is involved in biosynthesis of the thiamine precursor thiazole, in actinorhizal nodules of Alnus glutinosa. Plant $J$ 1996, 10:361-368.

22. Chatterjee A, Jurgenson CT, Schroeder FC, Ealick SE, Begley TP: Biosynthesis of thiamin thiazole in eukaryotes: conversion of NAD to an advanced intermediate. $J$ Am Chem Soc 2007, 129:2914-2922.

23. Chatterjee A, Schroeder FC, Jurgenson CT, Ealick SE, Begley TP: Biosynthesis of the thiamin-thiazole in eukaryotes: identification of a thiazole tautomer intermediate. $J$ Am Chem Soc 2008, 130:11394-11398.

24. Chatterjee A, Abeydeera ND, Bale S, Pai PJ, Dorrestein PC, Russell DH, Ealick SE, Begley TP: Saccharomyces cerevisiae THI4p is a suicide thiamine thiazole synthase. Nature 2011, 478:542-U146.

25. Rapala-Kozik M, Olczak M, Ostrowska K, Starosta A, Kozik A: Molecular characterization of the thi3 gene involved in thiamine biosynthesis in Zea mays: cDNA sequence and enzymatic and structural properties of the recombinant bifunctional protein with 4-amino-5-hydroxymethyl-2-methylpyrimidine (phosphate) kinase and thiamine monophosphate synthase activities. Biochem $J$ 2007, 408:149-159.

26. Ajjawi I, Tsegaye Y, Shintani D: Determination of the genetic, molecular, and biochemical basis of the Arabidopsis thaliana thiamin auxotroph $\boldsymbol{t h 1}$. Arch Biochem Biophys 2007, 459:107-114.

27. Hasnain G, Roje S, Sa N, Zallot R, Ziemak MJ, De Crecy-Lagard V, Gregory JF, Hanson AD: Bacterial and plant HAD enzymes catalyze a missing phosphatase step in thiamin diphosphate biosynthesis. Biochem J 2016, 473:157-166. 
28. Ajjawi I, Rodriguez Milla MA, Cushman J, Shintani DK: Thiamin pyrophosphokinase is required for thiamin cofactor activation in Arabidopsis. Plant Mol Biol 2007, 65:151162.

29. Frelin O, Agrimi G, Laera VL, Castegna A, Richardson LGL, Mullen RT, Lerma-Ortiz C, Palmieri F, Hanson AD: Identification of mitochondrial thiamin diphosphate carriers from Arabidopsis and maize. Functional \& Integrative Genomics 2012, 12:317-326.

30. Bocobza SE, Malitsky S, Araujo WL, Nunes-Nesi A, Meir S, Shapira M, Fernie AR, Aharoni A: Orchestration of thiamin biosynthesis and central metabolism by combined action of the thiamin pyrophosphate riboswitch and the circadian clock in Arabidopsis. Plant Cell 2013, 25:288-307.

31. Pourcel L, Moulin M, Fitzpatrick TB: Examining strategies to facilitate vitamin $\mathbf{B}_{1}$ biofortification of plants by genetic engineering. Frontiers in Plant Science 2013, 4.

32. Noctor G, Hager J, Li SC: Biosynthesis of NAD and its manipulation in plants. In Biosynthesis of vitamins in plants: Vitamins A, B1, B2, B3, B5, Pt A: Vitamins A, B1, B2, $B 3, B 5$. Edited by Rebeille F, Douce R. Academic Press Ltd-Elsevier Science Ltd; 2011:153-201.

33. Balmer Y, Koller A, del Val G, Manieri W, Schurmann P, Buchanan BB: Proteomics gives insight into the regulatory function of chloroplast thioredoxins. Proc Natl Acad Sci U S A 2003, 100:370-375.

34. Lemaire SD, Guillon B, Le Marechal P, Keryer E, Miginiac-Maslow M, Decottignies P: New thioredoxin targets in the unicellular photosynthetic eukaryote Chlamydomonas reinhardtii. Proc Natl Acad Sci U S A 2004, 101:7475-7480.

35. Fitzpatrick TB, Thore S: Complex behavior: from cannibalism to suicide in the vitamin B 1 biosynthesis world. Curr Opin Struct Biol 2014, 29:34-43.

36. Fenwick MK, Mehta AP, Zhang Y, Abdelwahed SH, Begley TP, Ealick SE: Non-canonical active site architecture of the radical SAM thiamin pyrimidine synthase. Nature Communications 2015, 6.

37. Shimamoto K, Nelson OE: Movement of ${ }^{14} \mathrm{C}$-compounds from maternal tissue into maize seeds grown in vitro Plant Physiol 1981, 67:429-432.

38. Martinis J, Gas-Pascual E, Szydlowski N, Crevecoeur M, Gisler A, Burkle L, Fitzpatrick TB: Long-distance transport of thiamine (vitamin $B_{1}$ ) is concomitant with that of polyamines. Plant Physiol 2016, 171:542-553.

-. $\quad$ This work identifies for the first time a transporter that mediates thiamin transport into the phloem and its function in shoot-to-root partitioning of thiamin.

39. Lerma-Ortiz C, Jeffryes JG, Cooper AJL, Niehaus TD, Thamm AMK, Frelin O, Aunins T, Fiehn O, de Crecy-Lagard V, Henry CS, et al.: 'Nothing of chemistry disappears in biology': the Top 30 damage-prone endogenous metabolites. Biochem Soc Trans 2016, 44:961-971.

- The authors combined information from the biochemical litterature, from cheminformatics, and from genome-scale metabolic models to define a 'Top 30' list of metabolites that are prone to chemical damage. Thiamin is included in this list. 
40. Hanson AD, Henry CS, Fiehn O, de Crecy-Lagard V: Metabolite damage and metabolite damage control in plants. In Annual Review of Plant Biology, Vol 67. Edited by Merchant SS. Annual Reviews; 2016:131-152.

- An excellent review on metabolite damage and metabolite damage control mechanisms in plants.

41. Linster CL, Van Schaftingen E, Hanson AD: Metabolite damage and its repair or preemption. Nature Chemical Biology 2013, 9:72-80.

42. Goyer A, Hasnain G, Frelin O, Ralat MA, Gregory JF, Hanson AD: A cross-kingdom Nudix enzyme that pre-empts damage in thiamin metabolism. Biochem J 2013, 454:533542.

43. Zallot R, Yazdani M, Goyer A, Ziemak MJ, Guan JC, McCarty DR, de Crecy-Lagard V, Gerdes S, Garrett TJ, Benach J, et al.: Salvage of the thiamin pyrimidine moiety by plant TenA proteins lacking an active-site cysteine. Biochem $J$ 2014, 463:145-155.

44. Yazdani M, Zallot R, Tunc-Ozdemir M, de Crecy-Lagard V, Shintani DK, Hanson AD: Identification of the thiamin salvage enzyme thiazole kinase in Arabidopsis and maize. Phytochemistry 2013, 94:68-73.

45. Mitsunaga T, Shimizu M, Iwashima A: Occurrence of thiamine-binding proteins in plant seeds. Journal of Plant Physiology 1986, 124:177-180.

46. Shimizu M, Mitsunaga T, Inaba K, Yoshida T, Iwashima A: Accumulation of thiamine and thiamine-binding protein during development of rice seed. Journal of Plant Physiology 1990, 137:123-124.

47. Mitsunaga T, Shimizu M, Iwashima A: A possible role for thiamine-binding protein in the germination of rice seed. Journal of Plant Physiology 1987, 130:279-284.

48. Watanabe K, Takahashi $\mathrm{H}$, Ampo M, Mitsunaga T: Change of thiamin-binding protein and thiamin levels during seed maturation and germination in sesame. Plant Physiology and Biochemistry 2003, 41:973-976.

49. Watanabe K, Nishida N, Adachi T, Ueda M, Mitsunaga T, Kawamura Y: Accumulation and degradation of thiamin-binding protein and level of thiamin in wheat seeds during seed maturation and germination. Bioscience Biotechnology and Biochemistry 2004, 68:1243-1248.

50. Nishino A, Nishino H, Iwashima A: Presence of a thiamine-binding protein in rice bran. $J$ Nutr Sci Vitaminol (Tokyo) 1980, 26:415-418.

51. Nishimura H, Uehara Y, Sempuku K, Iwashima A: Purification and some properties of thiamine-binding protein from rice bran. J Nutr Sci Vitaminol (Tokyo) 1984, 30:1-10.

52. Seaver SMD, Bradbury LMT, Frelin O, Zarecki R, Ruppin E, Hanson AD, Henry CS: Improved evidence-based genome-scale metabolic models for maize leaf, embryo, and endosperm. Frontiers in Plant Science 2015, 6.

- The authors developed a new evidence-based genome-scale reconstruction method to build organ specific metabolic model for the maize leaf, and tissue specific metabolic models for maize embryo and endosperm cells. 


\section{Figure captions}

Figure 1. Rice grain anatomy and model of thiamin synthesis, salvage, transport and storage in the rice grain. In the early stages of development, the endosperm, i.e. the starchy endosperm and the aleurone cell layer, expresses the thiamin biosynthetic genes THIC and THII, suggesting that thiamin is synthesized de novo in these tissues. In the late stages of development, the expression of THIC decreases while that of THII increases in the endosperm, as shown in maize [11], suggesting that the endosperm loses its capability to synthesize thiamin de novo but may still be able to produce thiamin by coupling de novo thiazole biosynthesis and pyrimidine salvage. Thiamin vitamers transported from maternal tissues to the endosperm may be a major source of thiamin for the endosperm, especially during the late stages of development. Thiamin in its free form can be stored in the aleurone cell layer by interaction with thiamin binding proteins (TBPs).

Figure 2. The de novo thiamin biosynthetic pathway in plants. Thiazole and pyrimidine synthesis branches are highlighted in green and gold, respectively. Thiamin biosynthetic enzymes and transporters are indicated in white font in grey boxes. ThDP-dependent enzymes are indicated in white font in blue boxes. The red line illustrates the negative regulation of HMPPP on ThMP synthase activity of TH1. TK, transketolase; DXPS, 1-deoxy-D-xylulose-5phosphate synthase; PDH, pyruvate dehydrogenase; AHAS, acetohydroxyacid synthase; PDC, pyruvate decarboxylase; KGDH, $\alpha$-ketoglutarate dehydrogenase; $\mathrm{BCKDH}$, branched-chain $\alpha$ ketoacid dehydrogenase. 


\section{Figure1}

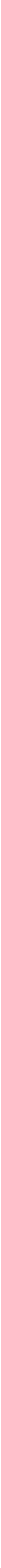


Figure2

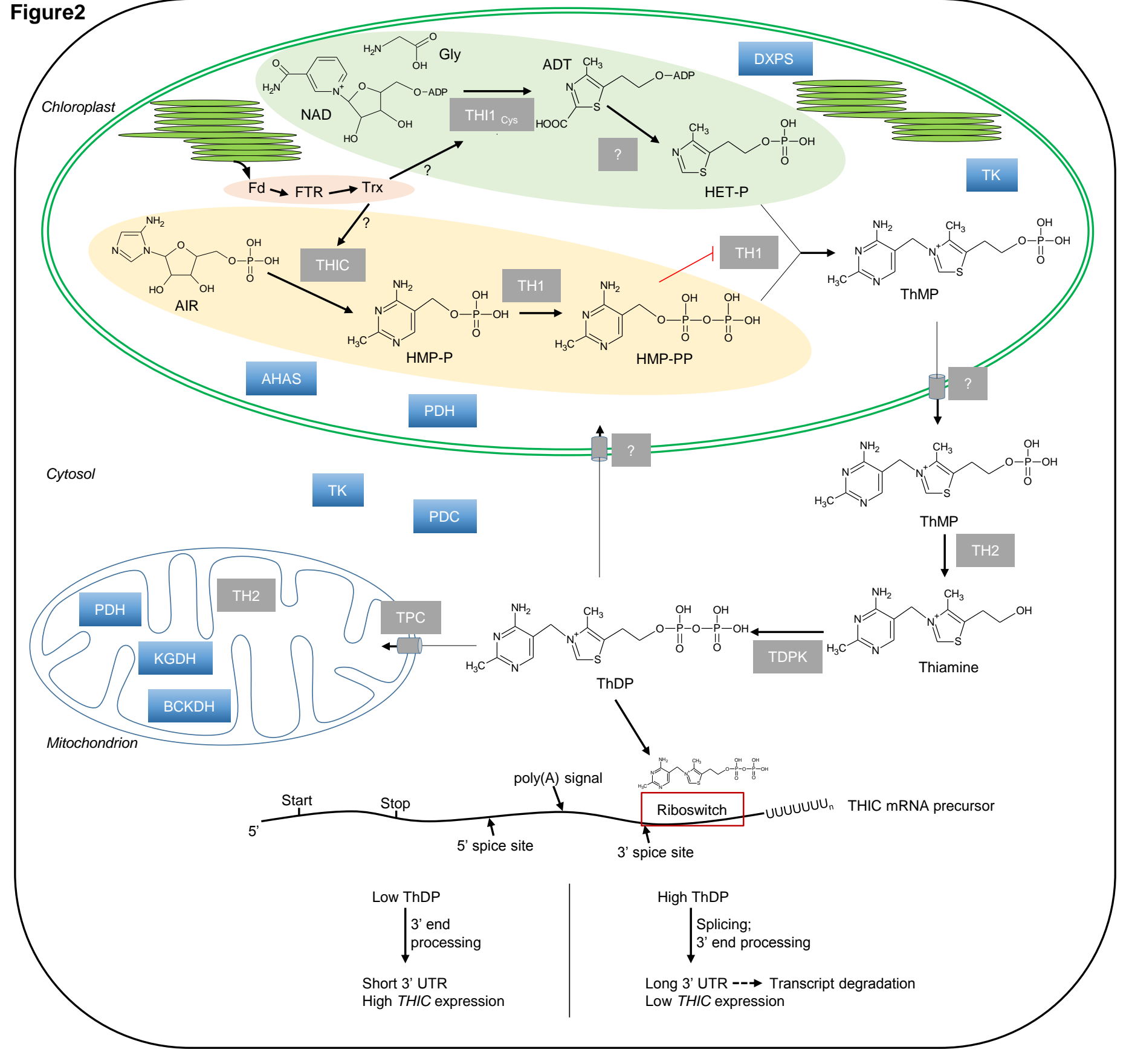

Short 3' UTR

High THIC expression
Long 3' UTR $\rightarrow$ Transcript degradation Low THIC expression 
Table 1. Maximum increase in total thiamin obtained by engineering the thiamin biosynthesis pathway in Arabidopsis and rice leaves and seeds. Fold increases are relative to non-engineered plants. n.m., not measured.

\begin{tabular}{llcccc}
\hline Organism & Organ & Thi1 & ThiC & Thi1 x ThiC & Reference \\
\hline \multirow{2}{*}{ Arabidopsis } & Leaves & 0 & 1.6 & 3.4 & {$[8,28]$} \\
& Seeds & 0 & 1.7 & 2.6 & \\
\hline \multirow{2}{*}{ Rice } & Leaves & 0 & 0 & 2.5 & {$[9]$} \\
& Unpolished seeds & 0 & 0 & 5 & \\
& Polished seeds & n.m. & n.m. & 1.3 & \\
\hline
\end{tabular}




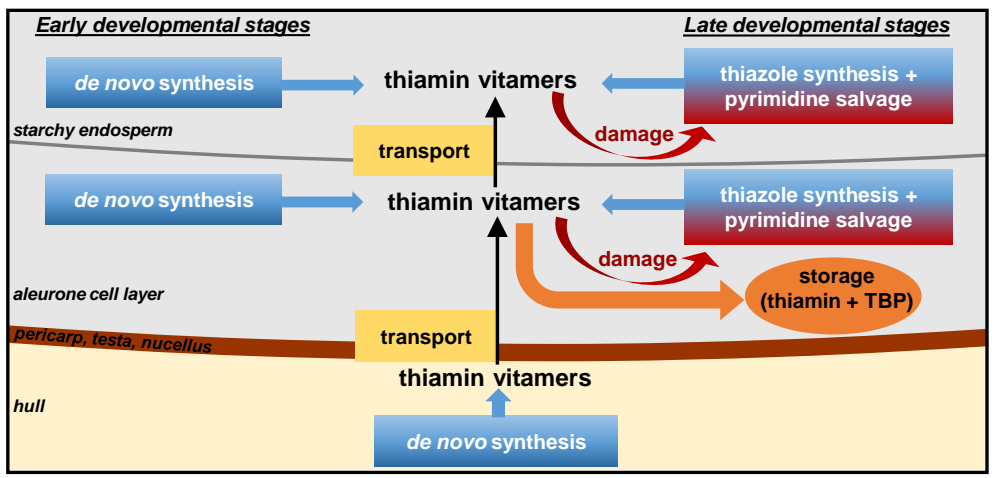

